OPEN ACCESS

Edited by:

Martin Craig Taylor,

University of London, United Kingdom

Reviewed by:

Manju Jain,

Central University of Punjab, India

Renato Augusto DaMatta,

State University of the North

Fluminense Darcy Ribeiro, Brazil

*Correspondence: Claudia Masini d'Avila-Levy

claudia.davila@ioc.fiocruz.br

Specialty section: This article was submitted to

Parasite and Host,

a section of the journal

Frontiers in Cellular and

Infection Microbiology

Received: 29 October 2021 Accepted: 17 January 2022

Published: 15 February 2022

Citation:

Boucinha C, Andrade-Neto W, Ennes-Vidal V, Branquinha MH, Santos ALS, Torres-Santos EC and d'Avila-Levy CM (2022)

A Stroll Through the History of Monoxenous Trypanosomatids Infection in Vertebrate Hosts. Front. Cell. Infect. Microbiol. 12:804707. doi: 10.3389/fcimb.2022.804707

\section{A Stroll Through the History of Monoxenous Trypanosomatids Infection in Vertebrate Hosts}

\author{
Carolina Boucinha ${ }^{1}$, Valter Viana Andrade-Neto ${ }^{1}$, Vítor Ennes-Vidal ${ }^{1}$, \\ Marta Helena Branquinha ${ }^{2}$, André Luis Souza dos Santos ${ }^{2}$, Eduardo Caio Torres-Santos ${ }^{1}$ \\ and Claudia Masini d'Avila-Levy ${ }^{1 *}$
}

${ }^{1}$ Instituto Oswaldo Cruz, Fundação Oswaldo Cruz (FIOCRUZ), Rio de Janeiro, Brazil, ${ }^{2}$ Instituto de Microbiologia Paulo de Góes, Universidade Federal do Rio de Janeiro, Rio de Janeiro, Brazil

The Trypanosomatidae family encompasses unicellular flagellates and obligate parasites of invertebrates, vertebrates, and plants. Trypanosomatids are traditionally divided into heteroxenous, characterized by the alternation of the life cycle between an insect vector and a plant or a vertebrate host, including humans being responsible for severe diseases; and monoxenous, which are presumably unique parasites of invertebrate hosts. Interestingly, studies reporting the occurrence of these monoxenous trypanosomatids in humans have been gradually increasing, either associated with Leishmania co-infection, or supposedly alone either in immunocompromised or even more sporadically in immunocompetent hosts. This review summarizes the first reports that raised the hypothesis that monoxenous trypanosomatids could be found in vertebrate hosts till the most current reports on the occurrence of Crithidia spp. alone in immunocompetent human patients.

Keywords: heteroxenous, immunocompromised hosts, monoxenic, pathogenicity, trypanosomatidae, immunocompetent host

\section{INTRODUCTION}

The family Trypanosomatidae encompasses eukaryotic flagellates, unicellular and obligatory parasites of invertebrates, vertebrates, and plants. The unique morphology of its single mitochondrion DNA (called kinetoplast) is an apomorphy of the Class Kinetoplastea, and its specific cellular positioning in relation to the nucleus and the point of flagella emersion allows the identification of specific life cycle forms, some of which are genus-specific (reviewed by d'Avila-Levy et al., 2015). Trypanosomatids are traditionally divided into heteroxenous, characterized by alternating the life cycle between an insect vector and a vertebrate host or a plant; and monoxenous, which are parasites presumably exclusive of invertebrate hosts, mainly insects. The heteroxenous trypanosomatids are the causative agents of severe human diseases that are mainly transmitted by an insect vector, such as Chagas disease (caused by Trypanosoma cruzi), sleeping sickness (caused by Trypanosoma brucei sensu lato), and the various forms of cutaneous and visceral leishmaniasis (caused by Leishmania spp.) (Vickerman, 1994). On the contrary to these pathogenic flagellates, the monoxenous (or "lower") trypanosomatids have received considerably less effort and attention by the scientific community since these parasites are found, a priori, only in the digestive 
tract of insects, and even its pathogenicity to insects is questionable, with few exceptions. Nevertheless, species typically non-pathogenic to humans are important models for understanding the biological behavior, biochemistry and molecular biology of pathogenic trypanosomatids (d'Avila-Levy et al., 2015). In addition, these organisms are being explored as vaccine candidates; for example, Phytomonas serpens confers protective immunity against T. cruzi (Pinge-Filho et al., 2005; da Silva et al., 2013); and as a platform to produce folded eukaryotic proteins, such as erythropoietin and insulin produced by Leishmania tarentolae (Dortay and MuellerRoeber, 2010). Finally, monoxenous trypanosomatids are attracting the attention of researchers in the field due to the increasing reports on the occurrence of these presumably nonpathogenic trypanosomatids in humans (Dedet et al., 1995; Jiménez et al., 1996; Pacheco et al., 1998; Miller, 2000; Ferreira and Borges, 2002; Chicharro and Alvar, 2003). Here, we will present a historical review since the first reports that raised the hypothesis that monoxenous trypanosomatids could be found in vertebrate hosts till the recent reports on the occurrence of Crithidia spp. in immunocompetent human patients.

\section{FIRST REPORTS ON THE OCCURRENCE OF MONOXENIC TRYPANOSOMATIDS IN HUMANS}

A comprehensive bibliographic survey on the biology and physiology of monoxenous trypanosomatids by McGhee and Cosgrove (1980) described a possible human infection attributed to the genus Herpetomonas. The authors reported a patient with undefined symptoms (such as fever and moments of unconsciousness) admitted to a hospital in Texas (USA). Several tests were performed, including a liver biopsy, which subsequently was inoculated in culture medium. After seven days, it was possible to observe the proliferation offlagellates. However, neither promastigote nor epimastigote forms were observed. The authors suggested that the flagellate found in the patient's sample was a species of the genus Herpetomonas based on the microscopic observations and the culture behavior, but the possibility of culture contamination by the culture medium manufacturer or by sample handling in the hospital was not ruled out (McGhee and Cosgrove, 1980). In addition, this report also mentioned evidence of Herpetomonas megaseliae (syn. Herpetomonas muscarum (Borghesan et al., 2013) infection in lizards and mice (Daggett et al., 1972; McGhee and Cosgrove, 1980) and species of Crithidia, Leptomonas, and Blastocrithidia that managed to grow in chicken embryos maintained at $37^{\circ} \mathrm{C}$ (Schmittner and Mcghee, 1970). However, the authors also stated that previous claims of pathogenicity of monoxenic trypanosomatids in infection of vertebrates were made but that none of these cases could be sustained after close examination. To sum up, McGhee \& Cosgrove challenged: "Although there is no proof of lower trypanosomatids infecting vertebrates, the possibility exists and should be considered by attending physicians and veterinarians" (McGhee and Cosgrove, 1980).
In 1991, an unusual clinical manifestation was reported in an immunosuppressed child in the Republic of Guinea-Bissau. The case drew physicians' attention, as it had symptoms caused by Leishmania species that cause visceral leishmaniasis. However, leishmaniasis had never been reported in the country by that moment. This led the researchers to suppose that the symptoms were caused by some unknown species of Leishmania or another opportunistic trypanosomatid present only in reptiles or small mammals, different from the well-known pathogenic species (Sabbatani et al., 1991).

Later, in 1995, a report on the island of Martinique (France) of a human immunodeficiency virus (HIV)-positive patient who developed the diffuse cutaneous nodular syndrome, usually caused by Leishmania species, also caught the attention of researchers (Dedet et al., 1995). The parasites isolated from the skin lesions were submitted to isoenzymes characterization and optical and electron microscopy. Interestingly, the parasite differed isoenzymatically from all known Leishmania species. The authors assumed that the parasite present in the lesion could be a monoxenous trypanosomatid since both microscopies revealed the presence of the kinetoplast and opisthomastigote stages (Dedet et al., 1995), and the successful infection could be related to the immunodeficiency of the patient. Some years later, Boisseau-Garsaud et al., 2000) reported a second case, but this time in a patient not affected by any immunodeficiency, in the same location (Martinique - France) presenting a localized skin lesion. The isoenzyme analysis revealed that this parasite showed the same isoenzymatic profile of the parasite described previously by Dedet and colleagues. As none of the two patients had left the island in their entire lives, the authors suggested that an insect parasitic monoxenous trypanosomatid occurring in that region could be infecting humans in a diffuse or localized manner depending on the health status of the host (Boisseau-Garsaud et al., 2000). However, two years later, Dedet research group submitted the two clinical isolates to gene sequencing. Following the isoenzymic characterization, both strains were identical to each other and distant from any other known Leishmania species, although related to L. enriettii. Thus, it was not possible to conclude whether these isolates would be grouped within Leishmania (Leishmania), Leishmania (Viannia) or if they should belong to a new clade within the euleishmania group (Noyes et al., 2002). Several years later, these isolates were recognized as $L$. martiniquensis, with a divergent taxonomic position, distinct from any other existing taxa responsible for an endemic focus of cutaneous leishmaniasis in Martinique (Desbois et al., 2014).

In 1996, Jiménez and colleagues reported a case in Madrid of a patient who, in addition to having immunosuppression, was an injecting drug user. This patient was hospitalized with an extensive medical history of infections and, among them, clinical suspicion of visceral leishmaniasis. Although it was not possible to observe amastigote forms in the bone marrow aspirate, some flagellates were isolated in culture, and promastigote forms were observed by optical microscopy. The isoenzymatic characterization revealed a pattern different from 
Leishmania reference strains, and the sample did not hybridize with Leishmania-specific probes. Moreover, isolated promastigotes could not infect either BALB/c mice or Syrian golden hamsters. Therefore, the authors ruled out the possibility of Leishmania infection and suggested that the patient could be infected by a monoxenous trypanosomatid that could cause leishmaniasis-like symptoms in HIV-positive patients. The contamination route could be syringe washing with water contaminated with parasitic insect feces (Jiménez et al., 1996).

In Brazil, Pacheco et al. (1998) identified amastigotes in a bone marrow aspiration from an HIV-positive patient with clinical symptoms of visceral leishmaniasis. The patient's place of residence is endemic for L. braziliensis, a species responsible for cutaneous leishmaniasis, which raised the hypothesis of visceralization of a typical cutaneous species. The parasite grew in culture as promastigotes and could not establish infection in mouse peritoneal macrophages, suggesting the nonpathogenicity of the flagellate (Pacheco et al., 1998). The isoenzyme analysis revealed that the isolated parasite did not correspond to all the assessed known genera, including the classical pathogenic ones. Southern blot hybridization technique revealed homology with the monoxenous species Leptomonas pulexsimulantis (generally parasitic species of dog fleas) (Pacheco et al., 1998). The authors assumed that the patient's immunosuppressed condition probably facilitated the establishment of opportunistic monoxenous trypanosomatids in the organism already weakened by HIV (Pacheco et al., 1998).

At the beginning of the 2000s, the progressive increase in reports of leishmaniasis symptoms in patients caused by nonleishmania trypanosomatids prompted Chicharro and Alvar (2003) to publish the first review on the topic - Monoxenous trypanosomatids in immunosuppressed patients. Although the pathogenicity of monoxenous trypanosomatids is debatable, the authors argued that these reports are not surprising since individuals severely immunocompromised because of HIV infection may also be more susceptible to other infections, including trypanosomatids, which are generally considered non-pathogenic.

In 2008, an immunosuppressed patient was admitted to a hospital in Montpellier (France), the symptoms being fever and persistent headache. The patient had been HIV-positive since 1991 and had a history of antiretroviral failure with low CD4+ T cell counts, leading to many opportunistic diseases. Among the exams performed, a blood sample was inoculated in an NNN medium. Four weeks later, kinetoplastid flagellates with morphology different from that of typical Leishmania promastigotes were isolated. The patient was discharged from the hospital and recovered spontaneously without any antileishmanial treatment. Subsequent attempts to re-isolate the parasite either from the blood or from the bone marrow were unsuccessful. Sequencing of the $5 S$ and $18 S$ ribosomal DNA region from the isolated parasite revealed genetic proximity to the monoxenous trypanosomatid Herpetomonas samuelpessoai (Morio et al., 2008). It seems clear that the patient's immune status can pave the road for opportunistic infections, like the monoxenic trypanosomatids. It is interesting to note that all these reports identified the later after culture isolation, which creates an important bias that will be later addressed.

\section{CO-INFECTIONS LEISHMANIA AND LEPTOMONAS}

Besides the possible infections by monoxenous trypanosomatids reported in immunosuppressed patients, another phenomenon has been attracting attention in the scientific community: the coinfection of monoxenous trypanosomatids, mainly of the genus Leptomonas, and Leishmania species. This co-infection has been reported increasingly, and most of these reports come from the Indian subcontinent, where visceral leishmaniasis or Kala-azar is frequent (Srivastava et al., 2010; Ghosh et al., 2012; Selvapandiyan et al., 2015; Thakur et al., 2020).

By screening 120 patients with kala-azar symptoms in the Indian subcontinent, Srivastava et al. (2010) obtained successful culture isolates from all splenic aspirates. The restriction fragment length polymorphisms (RFLP) of the Hsp70 gene from the isolates identified nine samples with a restriction pattern that differed from known Leishmania species. Furthermore, Hsp70 and $18 \mathrm{~S}$ gene sequencing of two isolates revealed a genetic relatedness to Leptomonas seymouri (Srivastava et al., 2010). This report is interesting because it identifies a potential monoxenic trypanosomatid in 9 different kala-azar patients from different regions of India with no history of HIV/AIDS. Although, as expected, BALB/c inoculation with $L$. donovani led to death after 20 days, while the BALB/c group inoculated with one of the atypical isolates survived and 45 days after infection, DNA was extracted, and the product of rDNAITS1 PCR analyzed by gel electrophoresis revealed a pattern consistent with Lept. seymouri and L. donovani (Srivastava et al., 2010). This raised concerns on the original culture: was it mixed with a higher proportion of Lept. seymouri that outnumbers but does not eliminate $L$. donovani in culture? Kala-azar causes a significant immunosuppression that could pave the road for Lept. seymouri infection; therefore, in mice, the few $L$. donovani cells would increase, but the inoculum would not be enough to establish a deadly infection. Two years later, another research group showed that Lept. seymouri was identified in four out of 29 DNA clinical samples (peripheral blood or skin biopsy) from patients with visceral leishmaniasis or post-kala-azar dermal leishmaniasis (PKDL). In addition, two out of seven parasites isolated in culture from blood samples revealed similarity to Lept. seymouri (Ghosh et al., 2012), which was first identified due to an atypical ITS1 PCR product, followed by DNA sequencing and phylogenetic analysis (Ghosh et al., 2012). Later, a screening by ITS1-RFLP of cutaneous leishmaniasis biopsies from 57 patients in a new endemic region in India revealed that $38.5 \%(22 / 57)$ of the samples presented two PCR products with a restriction fragment profile consistent with $L$. donovani and Lept. seymouri. Subsequently, the amplicons from representative samples $(n=9)$ of the possible co-infection biopsies were sequenced, revealing maximum identity with $L$. donovani and Lept. seymouri (Thakur et al., 2020). Considering, that the 
PCR was performed directly in the biopsies, this is an important piece of evidence towards the participation of monoxenic trypanosomatids in leishmaniasis physiopathology.

Usually, when patients manifest the typical clinical features of visceral leishmaniasis, PKDL or cutaneous leishmaniasis, the test performed to confirm the diagnosis is either the detection of the parasite in smears on a stained slide or culture positivity; in some cases, ELISA tests are also used. However, DNA sequencing is rarely performed to confirm the identity of the species found. This has led the authors to question whether all the parasites isolated from these patients with leishmaniasis may be infected with $L$. donovani and any potential co-infecting monoxenous trypanosomatid species, since they are not typed (Srivastava et al., 2010; Thakur et al., 2020). Interestingly, the authors stated that the detection of the nonpathogenic species in patients in the Indian sub-continent must be carefully considered: first, a more in-depth study on the clinical relevance and susceptibility of this parasite to infect humans is worth; second, considering that the co-infections were identified in an area where there are a relevant number of patients resistant to the antimonial used for the treatment of visceral leishmaniasis, this strain of Leptomonas could be more resistant to antimony or could act synergistically to $L$. donovani leading to treatment resistance (Ghosh et al., 2012).

In this same direction, Singh et al. (2013) using the next generation sequencing of samples collected from patients affected by visceral leishmaniasis in India, identified Lept. seymouri in co-infection with L. donovani. Although both species were in concomitance in splenic aspirates, it was not possible to attribute the pathogenicity of Leptomonas to the clinical cases. Furthermore, the two species have low genetic divergence and are morphologically similar: both have promastigote forms within the host insect. Thus, the researchers also believe that cases like this are underestimated and that the presence of Leptomonas or another monoxenous associated with the Leishmania species is greater than reported (Singh et al., 2013).

To evaluate the mechanisms that may favor the appearance of monoxenous trypanosomatids in vertebrate hosts, Kraeva et al. (2015) further investigated two of the clinical isolates of Lept. seymouri found in Singh's work (Singh et al., 2013). Although the isolates proved to be adapted to grow at high temperatures and they were able to remain in the digestive tract of the insect vector, Lept. seymouri was unable to establish an infection in mammalian macrophages in vitro. The authors concluded that despite the adaptations undergone by Lept. seymouri, its occurrence in a vertebrate host would only be possible under host immunosuppressant conditions. Those authors also point out that some DNA sequences deposited at Genbank as $L$. donovani are in fact Lept. seymouri and emphasized the importance of specific markers for identifying monoxenous species (Kraeva et al., 2015).

\section{CRITHIDIA OCCURRENCE IN IMMUNOCOMPETENT PATIENTS}

In 2019, Ghobakhloo and colleagues reported the occurrence of Crithidia either alone or in co-infection with L. major in human patients in Iran with no apparent immunosuppression. As expected, out of 167 patients screened, $92.8 \%$ had L. major as the only causative agent, while $5.4 \%$ accounted for co-infections, and in only $1.8 \%$ of the cases (4 patients), Crithidia was the sole agent. The study was focused on detecting insect trypanosomatids based on previous reports in Iran that provided preliminary evidence on the occurrence of insect trypanosomatids in immunocompetent patients. Considering the skepticism of the scientists on the occurrence of "monoxenous" trypanosomatids in healthy individuals and the general belief that all reports up to date are due to the culture strain/clone biased selection, the authors collected samples directly from the biopsies and performed microscopy and PCR amplification. Using two sets of gGAPDH primers, one that amplified Leishmania and the other Crithidia, the authors provided sequences that showed similarity to $C$. fasciculata reference sequences (Ghobakhloo et al., 2019). In addition to the molecular characterization of the clinical samples, the authors also analyzed some aspects of the isolates obtained in culture. The isolates were able to survive, even if less active and rounder, at a higher temperature that mimicked the temperature of the human body, in contrast to a C. fasciculata reference strain obtained from the Centre of Research in Infectious Disease, Laval University (Quebec, Canada). In addition, the clinical isolate of Crithidia was able to infect two macrophage cell lines (J774 and THP1). However, as expected, the infection index was lower when compared to L. infantum and L. major infection. Furthermore, the reference strain of Crithidia did not show the same behavior, it was unable to establish itself inside the macrophages (Ghobakhloo et al., 2019).

These reports open interesting discussions: for a time, the findings of monoxenous trypanosomatids in humans were associated with immunodeficiencies in the patient. In this work, some isolates of Crithidia were recovered from the lesions of immunocompetent patients. In addition, when LeishmaniaCrithidia co-infection was reported, the clinical cases were more severe when compared to patients who had only Leishmania isolated from the lesion. Another important observation is that, as described in the co-infection between Lept. seymouri and $L$. donovani (Ghosh et al., 2012), some of these co-infections did not respond to treatment with antimonial, which raised the question of the possible role of monoxenous trypanosomatids in resistance to the therapy (Ghobakhloo et al., 2019).

This article was refuted in the same year by Kostygov et al., 2019) who claimed that the methodologies used in Ghobakhloo's report were not sufficient to state that Crithidia was the sole agent causing skin damage in immunocompetent patients. Kostygov and colleagues claimed that the immune status of the patients was not assessed as well as the primer used to amplify gGAPDH from Leishmania could not amplify L. infantum (Kostygov et al., 2019). Subsequently, the authors replied that the Crithidia spp. were identified in patients with cutaneous leishmaniasis resistant to glucantime treatment, which led the researchers to a closer follow up of these patients. To this end, all patients were examined for immunodeficiency diseases, which were excluded, and then biopsies and smears from patients' lesions were collected under 
sterile conditions for parasite characterization. One sample was taken for culture, and three smears were prepared for microscopic study and PCR amplification. Regarding the PCR primer specificity, the authors replied that it could discriminate among L. infantum, $L$. major, and L. tropica (Motazedian, 2019). Concerning the specificity of the PCR primer designed to amplify Leishmania, the primer specific for Crithidia did amplify a fragment directly from the patients' smears. The amplicon was sequenced and revealed similarity to Crithidia. The same sample revealed only Crithidia in the cultures, subsequently identified in Dr. Marc Ouellette's research group (Laval University Research Center for Infectious Diseases, Quebec, Canada). Kostygov hypothesis would be that these four samples would be a co-infection between L. infantum and Crithidia, and the authors failed to detect the former by the PCR primers used. Then, as expected, in culture, the monoxenic counterpart outgrows the fastidious Leishmania.

In the same year, another report drew a lot of attention from the scientific community, also reaching the general press (Maruyama et al., 2019). The authors described one fatal case of a visceral leishmaniasis-like, being more severe and resistant to the treatment. The patient, which had a negative HIV diagnosis, died of disease and surgical complications. The parasite was isolated in culture from skin lesion and bone marrow. The isolated parasite is more closely related to C. fasciculata than to any Leishmania by several approaches, including whole-genome sequencing. After intravenous infection in female BALB/c mice with both isolates, only the bone marrow isolate was detected again in the liver and spleen; however, at significantly lower levels than the positive control (L. infantum), while the skin strain was detected at very low levels only in the liver. Therefore, those authors performed an artificial skin infection on the mice's ear, revealing that the skin strain caused lesions even more extensive than that resulting from the positive control (L. major) (Maruyama et al., 2019). Subsequently, Domagalska and Dujardin published a comment letter where they raised concerns to what we can call "environment biased selection hypothesis". The inoculation in the culture medium from the patient's biopsy can lead to the monoxenic counterpart prevailing in culture, although few Leishmania cells can remain. After that, in the mouse, the few Leishmania cells can outnumber the Crithidia. Therefore, a molecular identification after the experimental infection can help solve this puzzle (Domagalska and Dujardin, 2020). Finally, the last report sequencing the ITS1 gene directly from buffy coat samples in 14 patients (seven symptomatic and seven asymptomatic) in Iran revealed that one asymptomatic sample presented close similarity $(99.75 \%)$ to C. fasciulata (Rezaei et al., 2020).

\section{OCCURRENCE OF "MONOXENOUS" TRYPANOSOMATIDS IN NON-HUMAN MAMMALIAN HOSTS}

Human infection possibly caused by insect trypanosomatids calls the attention of the scientific community from the first report. However, not only humans are reported as possible unusual mammalian hosts for these trypanosomatids (Maslov et al., 2013). The possibility that insect trypanosomatids could be found in mammalian hosts started in the early description of these parasites, already by morphological observations (McGhee and Cosgrove, 1980). The first time, to our knowledge, that a DNA sequence was provided from monoxenic trypanosomatids isolated from mammals was from rodents and dogs in Egypt several years after the original isolation. The original parasite kept in culture for several years was sequenced and reported to be close to Herpetomonas (Podlipaev et al., 2004). A long jump in years and a more recent report performed DNA sequencing screening on 593 insectivorous bats from 8 species in the USA, revealing that $5(0.8 \%)$ were positive for Blastocrithidia spp. The incidence is not to be neglected since the positivity for T. cruzi, and $T$. dionisii were $0.17 \%$ and $1.5 \%$, respectively (Hodo et al., 2016). The screening was performed on DNA extracted from blood and heart tissue with no culture manipulation (Hodo et al., 2016). Another bat screening performed in Brazil revealed that out of 181 bat specimens from 18 species, one was found positive for a species belonging to the Crithidia genus, most likely $C$. mellificae (Rangel et al., 2019). The DNA amplification and sequencing were performed out of a culture isolate after rich medium cultivation (Rangel et al., 2019). In a following report from the same research group, a screening in 72 sylvatic animals captured in three distinct biomes in Brazil (Atlantic Forest, Cerrado, and Pantanal) revealed choanomastigote forms in 21 fresh hemoculture preparations (from marmosets, coatis, crabeating fox, and ocelots). Parasites could not be observed in direct blood examination (Dario et al., 2021). DNA extraction from these hemocultures and $18 S$ and $g G A P D H$ genes sequencing revealed a close similarity with C. mellificae (Dario et al., 2021).

The description of natural infections of monoxenous trypanosomatids in mammals has a tendency to increase. However, the actual parasite-host relationship is not fully established, not even if it is a parasite as classically defined. There are though several reports demonstrating successful in vitro infections by monoxenous trypanosomatids in humans and mouse cellular culture, as well as the successful growth of these parasites at $37{ }^{\circ} \mathrm{C}$ (Santos et al., 2004; Barreto-de-Souza et al., 2008; Matteoli et al., 2009; Pereira et al., 2010).

\section{FINAL CONSIDERATIONS}

The first report of a monoxenous trypanosomatid causing disease in mammals occurred as early as the 1980's. The reports are increasing in number and consistency. One should consider that for the general physician routine and patient handling and treatment, parasite isolation and identification is not necessary, and it is rarely performed. Therefore, the cases that resulted in a scientific report were either because Leishmania was not an occurring pathogen in the country or because the physicians' faced hurdles in the patient treatment, which required an "out of the box" approach. Anyway, the routine leishmaniasis diagnosis can never reveal the occurrence of monoxenic trypanosomatids: direct smears observation and 
immunoassays can reveal positivity for a monoxenic trypanosomatid without distinguishing from Leishmania. Isoenzymes and DNA sequencing can reveal the identity of the pathogen. However, the former requires parasite isolation in culture, while the latter can be performed directly in the patients' biopsy, but it is not frequently performed. Although it seems unequivocal that monoxenic trypanosomatids are occurring in wild mammals and humans and even in immunocompetent ones, the "environment biased selection hypothesis" is far from ruled out. Therefore, a co-infection monoxenic trypanosomatids/ Leishmania can be more common than imagined; the subsequent culture inoculation can favor the fast-growing monoxenic counterpart, which outgrows Leishmania without completely eliminating it from the culture media. Then, a mice infection can promote the selection in the other direction. Therefore, in order to take steps ahead on this intriguing pathway that could completely change our understanding of the trypanosomatids life cycle, it is critical that DNA amplification and sequencing is performed in the original biopsy, in the cultured sample, and then upon parasite isolated after the experimental infection. Nevertheless, it is time to move forward. The occurrence of monoxenic trypanosomatids in mammals is still viewed with suspicion by the scientific community. Several published reports needed to justify that there was no misidentification or contamination during the handling of the material, and primer specificity is questioned. Extensive screening of clinical samples by direct DNA sequencing would shed some light on the frequency of monoxenic trypanosomatids in humans and, more importantly, provide further evidence on their relevance in treatment resistance. Subsequently, further questions arise: how do they reach the lesion? As a secondary infection by a fly or as direct inoculation by a vector? The questions that arise are

\section{REFERENCES}

Barreto-de-Souza, V., Xavier Medeiros, T., Machado Motta, M. C., Bou-Habib, D. C., and Saraiva, E. M. (2008). HIV-1 Infection and HIV-1 Tat Protein Permit the Survival and Replication of a Non-Pathogenic Trypanosomatid in Macrophages Through TGF-Betal Production. Microbes Infect 10, 642-649. doi: 10.1016/j.micinf.2008.02.014

Boisseau-Garsaud, A. M., Cales-Quist, D., Desbois, N., Jouaunelle, J., Jouannelle, A., Pratlong, F., et al. (2000). A New Case of Cutaneous Infection by a Presumed Monoxenous Trypanosomatid in the Island of Martinique (French West Indies). Trans. R. Soc. Trop. Med. Hygiene 94, 51-52. doi: 10.1016/s0035-9203(00)90435-8

Borghesan, T. C., Ferreira, R. C., Takata, C. S. A., Campaner, M., Borda, C. C., Paiva, F., et al. (2013). Molecular Phylogenetic Redefinition of Herpetomonas (Kinetoplastea, Trypanosomatidae), a Genus of Insect Parasites Associated With Flies. Protist 164, 129-152. doi: 10.1016/j.protis.2012.06.001

Chicharro, C., and Alvar, J. (2003). Lower Trypanosomatids in HIV/AIDS Patients. Ann. Trop. Med. Parasitol. 97, 75-78. doi: 10.1179/000349803225002552

Daggett, P. M., Dollahon, N., and Janovy, J. (1972). Herpetomonas Megaseliae Sp. N. (Protozoa: Trypanosomatidae) From Megaselia Scalaris (Loe) Schmit (Diptera: Phoridae). J. Parasitol. 58, 946-949. doi: 10.2307/3286591

Dario, M. A., Lisboa, C. V., Silva, M. V., Herrera, H. M., Rocha, F. L., Furtado, M. C., et al. (2021). Crithidia Mellificae Infection in Different Mammalian Species in Brazil. Int. J. Parasitol.: Parasites Wildlife 15, 58-69. doi: 10.1016/ j.ijppaw.2021.04.003

da Silva, R., Malvezi, A. D., da Augusto, L. S., Kian, D., Tatakihara, V. L. H., Yamauchi, L. M., et al. (2013). Oral Exposure to Phytomonas Serpens endless, but the first and urgent approach is - are they alone or in a co-infection with Leishmania? A detailed study using the powerful DNA sequencing tools available today may provide a significant increase in knowledge about the possible role of monoxenous trypanosomatids in the pathogenic process, either alone or in co-infection with traditional pathogenic species.

\section{AUTHOR CONTRIBUTIONS}

CMDL and CBM wrote the first version of the manuscript. All the authors discussed, conceived and revised the manuscript. All authors contributed to the article and approved the submitted version.

\section{FUNDING}

We thank the Brazilian grant agencies Coordenação de Aperfeiçoamento de Pessoal de Nível Superior (CAPES), Conselho Nacional de Desenvolvimento Científico e Tecnológico (MCT/CNPq), Fundação Carlos Chagas Filho de Amparo à Pesquisa do Estado do Rio de Janeiro (FAPERJ) for funding, as well as Fundação Oswaldo Cruz (FIOCRUZ) for research infrastructure.

\section{ACKNOWLEDGMENTS}

We would like to thank all the members of our laboratories for helpful and stimulating discussions. We are thankful to all researchers that contributed to enrich COLPROT catalogue.

Attenuates Thrombocytopenia and Leukopenia During Acute Infection With Trypanosoma Cruzi. PloS One 8, e68299. doi: 10.1371/journal.pone.0068299

d'Avila-Levy, C. M., Boucinha, C., Kostygov, A., Santos, H. L. C., Morelli, K. A., Grybchuk-Ieremenko, A., et al. (2015). Exploring the Environmental Diversity of Kinetoplastid Flagellates in the High-Throughput DNA Sequencing Era. Memorias do Inst Oswaldo Cruz 110, 956-965. doi: 10.1590/0074-02760150253

Dedet, J. P., Roche, B., Pratlong, F., Caies-Quist, D., Jouannelle, J., Benichou, J. C., et al. (1995). Diffuse Cutaneous Infection Caused by a Presumed Monoxenous Trypanosomatid in a Patient Infected With HIV. Trans. R. Soc. Trop. Med. Hygiene 89, 644-646. doi: 10.1016/0035-9203(95)90427-1

Desbois, N., Pratlong, F., Quist, D., and Dedet, J. (2014). Leishmania (Leishmania) Martiniquensis N. Sp. (Kinetoplastida: Trypanosomatidae), Description of the Parasite Responsible for Cutaneous Leishmaniasis in Martinique Island (French West Indies). Parasite 21, 1-4. doi: 10.1051/parasite/2014011

Domagalska, M. A., and Dujardin, J. (2020). Non-Leishmania Parasite in Fatal Visceral Leishmaniasis-Like Disease, Brazil. Emerg. Infect. Dis. 26, 388. doi: 10.3201/eid2602.191428

Dortay, H., and Mueller-Roeber, B. (2010). A Highly Efficient Pipeline for Protein Expression in Leishmania Tarentolae Using Infrared Fluorescence Protein as Marker. Microbial Cell Factories 9, 1-10. doi: 10.1186/1475-2859-9-29

Ferreira, M. S., and Borges, A. S. (2002). Some Aspects of Protozoan Infections in Immunocompromised Patients - A Review. Memorias do Inst Oswaldo Cruz 97, 443-457. doi: 10.1590/S0074-02762002000400001

Ghobakhloo, N., Motazedian, M. H., Naderi, S., and Ebrahimi, S. (2019). Isolation of Crithidia Spp. From Lesions of Immunocompetent Patients With Suspected Cutaneous Leishmaniasis in Iran. Trop. Med. Int. Health 24, 116-126. doi: $10.1111 /$ tmi.13042 
Ghosh, S., Banerjee, P., Sarkar, A., Datta, S., and Chatterjee, M. (2012). Coinfection of Leptomonas Seymouri and Leishmania Donovani in Indian Leishmaniasis. J. Clin. Microbiol. 50, 2774-2778. doi: 10.1128/JCM.00966-12

Hodo, C. L., Goodwinb, C. C., Mayesc, B. C., Mariscald, ,.J. A., Waldrupd, K. A., and Hamer, S. A. (2016). Trypanosome Species, Including Trypanosoma Cruzi, in Sylvatic and Peridomestic Bats of Texas, USA. Acta Trop. 164, 259-266. doi: 10.1016/j.actatropica.2016.09.013

Jiménez, M. I., López-Vélez, R., Molina, R., Cañavate, C., and Alvar, J. (1996). HIV Co-Infection With a Currently Non-Pathogenic Flagellate. Lancet 347, 264265. doi: 10.1016/s0140-6736(96)90441-9

Kostygov, A. Y., Butenko, A., and Yurchenko, V. (2019). On Monoxenous Trypanosomatids From Lesions of Immunocompetent Patients With Suspected Cutaneous Leishmaniasis in Iran. Trop. Med. Int. Health 24, 127128. doi: 10.1111/tmi.13168

Kraeva, N., Butenko, A., Hlaváčová, J., Kostygov, A., Myškova, J., Grybchuk, D., et al. (2015). Leptomonas Seymouri: Adaptations to the Dixenous Life Cycle Analyzed by Genome Sequencing, Transcriptome Profiling and Co-Infection With Leishmania Donovani. PloS Pathog. 11, 1-23. doi: 10.1371/ journal.ppat.1005127

Maruyama, S. R., de Santana, A. K. M., Takamiya, N. T., Takahashi, T. Y., Rogerio, L. A., Oliveira, C. A. B., et al. (2019). Non-Leishmania Parasite in Fatal Visceral Leishmaniasis-Like Disease, Brazil. Emerg. Infect. Dis. 25, 2088-2092. doi: 10.3201/eid2511.181548

Maslov, D. A., Votýpka, J., Yurchenko, V., and Lukeš, J. (2013). Diversity and Phylogeny of Insect Trypanosomatids: All That is Hidden Shall be Revealed. Trends Parasitol 29, 43-52. doi: 10.1016/j.pt.2012.11.001

Matteoli, F. P., d'Avila-Levy, C. M., Santos, L. O., Barbosa, G. M., Holandino, C., Branquinha, M. H., et al. (2009). Roles of the Endosymbiont and Leishmanolysin-Like Molecules Expressed by Crithidia Deanei in the Interaction With Mammalian Fibroblasts. Exp. Parasitol. 121, 246-253. doi: 10.1016/j.exppara.2008.11.011

McGhee, R. B., and Cosgrove, W. B. (1980). Biology and Physiology of the Lower Trypanosomatidae. Microbiol. Rev. 44, 140-173. doi: 10.1128/mmbr.44.1.140173.1980

Miller, R. F. (2000). Clinical Presentation and Significance of Emerging Opportunistic Infections. J. Eukaryotic Microbiol. 47, 21-23. doi: 10.1111/ j.1550-7408.2000.tb00005.x

Morio, F., Reynes, J., Dollet, M., Pratlong, F., Dedet, J. P., and Ravel, C. (2008). Isolation of a Protozoan Parasite Genetically Related to the Insect Trypanosomatid Herpetomonas Samuelpessoai From a Human Immunodeficiency Virus-Positive Patient. J. Clin. Microbiol. 46, 3845-3847. doi: 10.1128/JCM.01098-08

Motazedian, M. H. (2019). Letter to the Editor Authors' Response. Trop. Med. Int. Health 24, 129. doi: 10.1111/tmi.13174

Noyes, H., Pratlong, F., Chance, M., Ellis, J., Lanotte, G., and Dedet, J. P. (2002). A Previously Unclassified Trypanosomatid Responsible for Human Cutaneous Lesions in Martinique (French West Indies) Is the Most Divergent Member of the Genus Leishmania. Parasitol. 124, 17-24. doi: 10.1017/S0031182001008927

Pacheco, R. S., Marzochi, M. C., Pires, M. Q., Brito, C. M., Madeira, M. D. F., and Barbosa-Santos, E. G. (1998). Parasite Genotypically Related to a Monoxenous Trypanosomatid of Dog's Flea Causing Opportunistic Infection in an HIV Positive Patient. Memórias do Inst Oswaldo Cruz 93, 531-537. doi: 10.1590/ s0074-02761998000400021

Pereira, F. M., Santos-Mallet, J. R., Branquinha, M. H., D’Avila-Levy, C. M., and Santos, A. L. S. (2010). Influence of Leishmanolysin-Like Molecules of Herpetomonas Samuelpessoai on the Interaction With Macrophages. Microbes Infect 12, 1061-1070. doi: 10.1016/j.micinf.2010.07.010

Pinge-Filho, P., Peron, J. P. S., de Moura, T. R., Menolli, R. A., Graça, V. K., Estevão, D., et al. (2005). Protective Immunity Against Trypanosoma Cruzi
Provided by Oral Immunization With Phytomonas Serpens: Role of Nitric Oxide. Immunol. Lett. 96, 283-290. doi: 10.1016/j.imlet.2004.09.010

Podlipaev, S. A., Sturm, N. R., Fiala, I., Fernandes, O., Westenberger, S. J., Dollet, M., et al. (2004). Diversity of Insect Trypanosomatids Assessed From the Spliced Leader. J. Eukaryotic Microbiol. Eukaryot Microbiol. 51, 283-290. doi: 10.1111/j.1550-7408.2004.tb00568.x

Rangel, D. A., Lisboa, C. V., Novaes, R. L. M., Silva, B. A., Souza, R., de, F., et al. (2019). Isolation and Characterization of Trypanosomatids, Including Crithidia Mellificae, in Bats From the Atlantic Forest of Rio De Janeiro, Brazil. PloS Negl. Trop. Dis. 13, 1-17. doi: 10.1371/journal.pntd.0007527

Rezaei, Z., Azarang, E., Shahabi, S., Omidian, M., Pourabbas, B., and Sarkari, B. (2020). Leishmania ITS1 Is Genetically Divergent in Asymptomatic and Symptomatic Visceral Leishmaniasis: Results of a Study in Southern Iran. J. Trop. Med. 2020, 1-7. doi: 10.1155/2020/5351098

Sabbatani, S., Isulerdo Calzado, A., Ferro, A., Lopez Goudiaby, A. M., Borghi, V., Zanchetta, G. P., et al. (1991). Atypical Leishmaniasis in an HIV-2-Seropositive Patient From Guinea-Bissau. AIDS 5, 899-901. doi: 10.1097/00002030199107000-00020

Santos, D. O., Bourguignon, S. C., Castro, H. C., Silva, J. S., Franco, L. S., Hespanhol, R., et al. (2004). Infection of Mouse Dermal Fibroblasts by the Monoxenous Trypanosomatid Protozoa Crithidia Deanei and Herpetomonas Roitmani. J. Eukaryotic Microbiol. 51, 570-574. doi: 10.1111/j.1550-7408.2004.tb00293.x

Schmittner, S. M., and Mcghee, R. B. (1970). Host Specificity of Various Species of Crithidia Léger. J. Parasitol. 56, 684-693. doi: 10.2307/3277713

Selvapandiyan, A., Ahuja, K., Puri, N., and Krishnan, A. (2015). Implications of Co-Infection of Leptomonas in Visceral Leishmaniasis in India. Parasitology 142, 1657-1662. doi: 10.1017/S0031182015001389

Singh, N., Chikara, S., and Sundar, S. (2013). SOLiD ${ }^{\mathrm{TM}}$ Sequencing of Genomes of Clinical Isolates of Leishmania Donovani From India Confirm Leptomonas Co-Infection and Raise Some Key Questions. PloS One 8, e55738. doi: 10.1371/ journal.pone.0055738

Srivastava, P., Prajapati, V. K., Vanaerschot, M., van der Auwera, G., Dujardin, J. C., and Sundar, S. (2010). Detection of Leptomonas Sp. Parasites in Clinical Isolates of Kala- Azar Patients From India. Infect. Genet. Evol. 10, 1145-1150. doi: 10.1016/j.meegid.2010.07.009

Thakur, L., Kushwaha, H. R., Negi, A., Jain, A., and Jain, M. (2020). Leptomonas Seymouri Co-Infection in Cutaneous Leishmaniasis Cases Caused by Leishmania Donovani From Himachal Pradesh, India. Front. Cell Infect. Microbiol. 10. doi: 10.3389/fcimb.2020.00345

Vickerman, K. (1994). The Evolutionary Expansion of the Trypanosomatid Flagellates. Int. J. Parasitol. 24, 1317-1331. doi: 10.1016/0020-7519(94)90198-8

Conflict of Interest: The authors declare that the research was conducted in the absence of any commercial or financial relationships that could be construed as a potential conflict of interest.

Publisher's Note: All claims expressed in this article are solely those of the authors and do not necessarily represent those of their affiliated organizations, or those of the publisher, the editors and the reviewers. Any product that may be evaluated in this article, or claim that may be made by its manufacturer, is not guaranteed or endorsed by the publisher.

Copyright (C) 2022 Boucinha, Andrade-Neto, Ennes-Vidal, Branquinha, Santos, Torres-Santos and d'Avila-Levy. This is an open-access article distributed under the terms of the Creative Commons Attribution License (CC BY). The use, distribution or reproduction in other forums is permitted, provided the original author(s) and the copyright owner(s) are credited and that the original publication in this journal is cited, in accordance with accepted academic practice. No use, distribution or reproduction is permitted which does not comply with these terms. 